\title{
Near infra-red spectroscopy of the asteroid 21 Lutetia
}

\section{Rotationally resolved spectroscopy of the surface}

\author{
D. A. Nedelcu ${ }^{1,2}$, M. Birlan ${ }^{1}$, P. Vernazza ${ }^{3}$, P. Descamps ${ }^{1}$, R. P. Binzel ${ }^{4}$, F. Colas ${ }^{1}$, A. Kryszczynska ${ }^{5}$, and S. J. Bus ${ }^{6}$ \\ ${ }^{1}$ Institut de Mécanique Céleste et de Calcul des Éphémérides (IMCCE), Observatoire de Paris, 77 avenue Denfert-Rochereau, \\ 75014 Paris Cedex, France \\ e-mail: [Mirel.Birlan; Pascal.Descamps;Francois.Colas]@imcce.fr \\ 2 Astronomical Institute of the Romanian Academy, 5 Cu titul de Argint, 75212 Bucharest, Romania \\ e-mail: nedelcu@imcce.fr \\ ${ }^{3}$ LESIA, Observatoire de Paris-Meudon, 5 place Jules Janssen, 92195 Meudon Cedex, France \\ e-mail: Pierre.Vernazza@obspm.fr \\ 4 Massachusetts Institute of Technology, 77 Massachusetts Avenue, Cambridge MA 02139, USA \\ e-mail: rpb@mit.edu \\ 5 Astronomical Observatory, Adam Mickiewicz University, Sloneczna 36, 60-286 Poznan, Poland \\ e-mail: agn@amu.edu.pl \\ ${ }^{6}$ Institute for Astronomy, 640 North A'ohouku Place, Hilo, HI 96720, USA \\ e-mail: sjb@ifa.hawaii.edu
}

Received 15 December 2006 / Accepted 16 April 2007

\section{ABSTRACT}

\begin{abstract}
Aims. In the framework of the ground-based science campaign dedicated to the encounter with the Rosetta spacecraft, the mineralogy of the asteroid (21) Lutetia was investigated.

Methods. Near-infrared (NIR) spectra of the asteroid in the $0.8-2.5 \mu \mathrm{m}$ spectral range were obtained with SpeX/IRTF in remote observing mode from Meudon, France in March and April 2006. We analysed these data together with previously acquired spectra - March 2003, August 2004. I-band relative photometric data obtained on 20 January 2006 using the $105 \mathrm{~cm}$ telescope from Pic $\mathrm{du}$ Midi, France has been used to build the ephemeris for physical observations. A $\chi^{2}$ test using meteorite spectra from the RELAB database was performed in order to find the best fit of complete visible + infrared (VNIR) spectra of Lutetia.

Results. The new spectra reveal no absorption features. We find a clear spectral variation (slope), and a good correspondence between spectral variations and rotational phase. Two of the most different spectra correspond to two opposite sides of the asteroid (sub-Earth longitude difference around $180^{\circ}$ ). For the neutral spectra a carbonaceous chondrite spectrum yields the best fit, while for those with a slightly positive slope the enstatitic chondrite spectra are the best analog. Based on the chosen subset of the meteorite samples, our analysis suggests a primitive, chondritic nature for (21) Lutetia. Differences in spectra are interpreted in terms of the coexistence of several lithologies on the surface where the aqueous alteration played an important role.
\end{abstract}

Key words. minor planets - asteroids - techniques: spectroscopic - methods: observational

\section{Introduction}

The ESA's flagship Rosetta spacecraft designed to investigate the comet 67P/Churyumov - Gerasimenko, successfully launched on March 2nd 2004, will include two asteroid flybys - (2867) Steins on September 2008, and (21) Lutetia on July 2010. Located in the inner part of the main belt, in an orbit with low eccentricity and inclination $(a=2.43489811, e=0.16380387$, $i=3$.064298), (21) Lutetia is the largest body among the mission's targets. Its diameter was estimated at $98.3 \pm 5.9 \mathrm{~km}$ by Mueller et al. (2006) and at $95.5 \pm 4.1 \mathrm{~km}$ by Tedesco et al. (1992) which allows direct measurement of the mass and density using the on-board radio science experiment.

Using ECAS data (Zellner et al. 1985), (21) Lutetia was classified by Barucci et al. (1987) and Tholen (1989) as an M-type because of its high IRAS albedo $(0.221 \pm 0.020)$ and was considered to have a metallic composition. Radio investigations of the asteroid show a low value of the radar albedo $(0.17 \pm 0.07)$, more typical to C-type rather than those of M-type asteroids
(Magri et al. 1999). The taxonomy study of Howell et al. (1994) by means of 52-color survey data, using the neural network technique, found that 21 Lutetia is more akin to the C-type asteroids than to the M-tye ones. The albedo inferred from polarimetry (Zellner et al. 1977; Lupishko \& Mohamed 1996) has a low value around 0.1 , far from the values derived in thermal domain. In the SMASSII feature-based taxonomy, Bus \& Binzel (2002) assign Lutetia to the the newly proposed $\mathrm{X}_{k}$-type considered as intermediary between X-core type and K-type asteroids.

More recent observations (Birlan et al. 2004, 2006) in the range $0.9-2.5 \mu \mathrm{m}$ showed a flat spectrum with a shallow band around $1 \mu \mathrm{m}$ and an overall neutral trend similar with the CV3 meteorite Vigarano. Another important spectral region is the $3 \mu \mathrm{m}$ band, associated with the presence of hydrated minerals to the asteroid surface. In the spectrophotometry survey of M-type asteroids by Rivkin et al. (2000), (21) Lutetia revealed a shallow absorption band in this region. This result was reinforced by spectroscopic investigations (Birlan et al. 2006) adding evidence of a primitive surface composition. 
Groundbased science results on (21) Lutetia bring forth some inconsistencies. The carbonaceous-chondrite analogy and the affinity for C-type remains incompatible with the high IRAS albedo value reconfirmed by Mueller et al. (2006) from thermalinfrared spectrophotometric measurements and thermal modeling $\left(p_{V}=0.208 \pm 0.025\right)$. Interestingly, Lazzarin et al. (2004) found in the $0.38-0.95 \mu \mathrm{m}$ a rather flat spectrum unlike those obtained by Bus (1999), Carvano et al. (2003), and Barucci et al. (2005) and two main absorption bands around $0.43 \mu \mathrm{m}$ and $0.51 \mu \mathrm{m}$, features not reported before. Variegation of spectral features with rotational phase and sub-Earth coordinates was then proposed as an explanation for these contradictory results. From series of spectra in the visible domain, Prokof'eva et al. (2005) found a variation with rotational phase in the width of $0.43 \mu \mathrm{m}$ band associated with hydrosilicates and explained it as a heterogeneous distribution of hydrated materials on the surface of Lutetia.

We present NIR spectra of the asteroid (21) Lutetia in the $0.8-2.5 \mu \mathrm{m}$ spectral range obtained using SpeX/IRTF instrument/telescope in March and April 2006. Further, our latest photometric observations in the visible region ( $I$-filter) were used to construct the physical ephemeris of (21) Lutetia. These ephemerides allowed us to link our NIR spectra, obtained in the period 2003-2006, with their corresponding regions on the asteroid surface. Finally, a comparison of our spectra with meteorites one (RELAB database) has been performed using the $\chi^{2}$ fitting test.

\section{Observations}

\subsection{Photometry}

Partial and complete lightcurves of (21) Lutetia were reported by several authors (Zappala et al. 1984; Lupishko \& Velichko 1987; Dotto et al. 1992; Lagerkvist et al. 1995) with amplitudes ranging from 0.1 to $0.25 \mathrm{mag}$. Using the inversion method for 32 lightcurves from 1962-1998 time interval, Torppa et al. (2003) constructed a shape model of Lutetia with some sharp and irregular features and rough global dimensions of $a / b=1.2$ and $b / c=1.2$ (where $a, b$, and $c$ are the semi-major axis of the ellipsoid figure, the closest to the shape model). The J2000.0 ecliptic coordinates of the adopted pole solutions for physical model are $\lambda_{1}=39^{\circ} \pm 10^{\circ}, \beta_{1}=3^{\circ} \pm 10^{\circ}$ and $\lambda_{2}=220^{\circ} \pm 10^{\circ}, \beta_{2}=3^{\circ} \pm 10^{\circ}$; the first solution is preferred to the second one (Mueller et al. 2006 and references within). The rotational period inferred from the 36-year baseline was derived to be $P=8.165455 \mathrm{~h}$ (Torppa et al. 2003).

Following Torppa et al. (2003)'s pole solution, (21) Lutetia was in a near equatorial aspect during the 2006 opposition. Thus our expectation was to observe a lightcurve with a relatively large amplitude.

We observed the asteroid on 20th of January 2006 using the $105 \mathrm{~cm}$ Cassegrain reflector at Pic du Midi, France equipped with $388 \times 284$ Thomson THX 7863 CCD camera, and the pixel scale of $0.50^{\prime \prime} /$ pixel. After the flat field correction with the ASTROL package the asteroid brightness was measured with PHOTOM aperture photometry programme included in the CCLRC package. The complete lightcurve in the $I$-band is presented in Fig. 1 and shows two asymmetric minima with an amplitude of 0.27 .

We conventionally define the planetocentric prime meridian of (21) Lutetia as the plane including the geocentric line of sight at the time of first lightcurve's minimum.

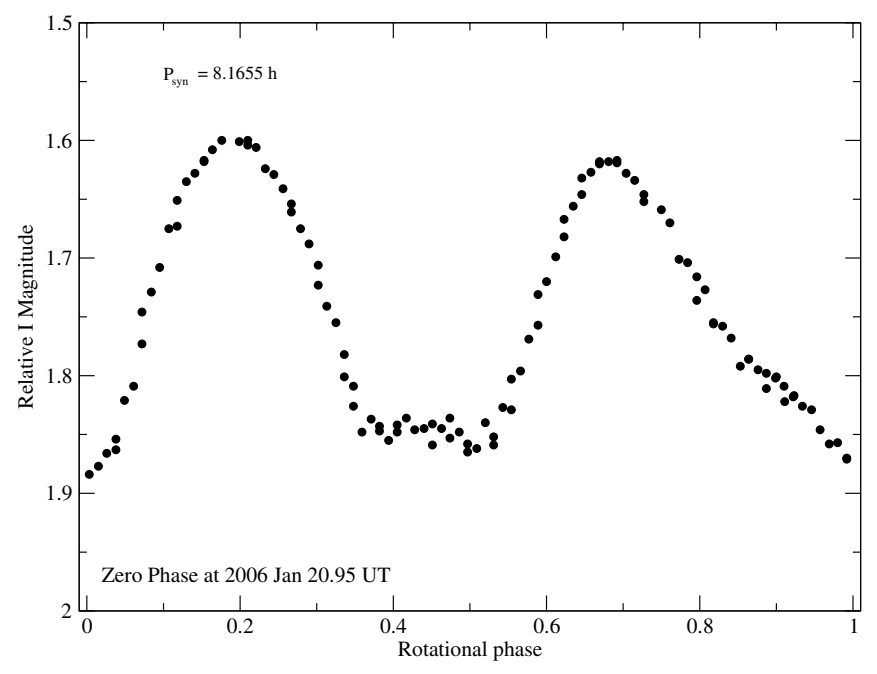

Fig. 1. Lightcurve of (21) Lutetia obtained at Pic du Midi in 20/21 January 2006. The 0 rotational phase was chosen to occur at $\mathrm{JD}=2453756.45$ and it determines the planetocentric prime meridian of the asteroid used for the work presented here. The lightcurve has an amplitude of 0.27 and presents two asymmetric minima.

Having the ephemeris for physical observations of the asteroid build from i) the preferred pole solution, ii) the rotation period and iii) the defined prime meridian, we can now establish a link between the spectra and the geometry of the observations. The eventual variations in the asteroids's spectra could be linked not only with changes in the sub-Earth latitude, but also with differences in sub-Earth longitudes from one spectrum to another. This effect is expected to be more important in the case of near-equatorial sub-Earth latitudes, than in the pole-on configuration when almost the same surface is presented during asteroid's rotation.

The error budget of this analysis is dominated by the uncertainty in the spin vector orientation which equally affects both the planetocentric latitude and longitude. The second order largest contribution in the error arises from the estimation of the ellipsoid flatness from the asteroid shape model axis ratio (Torppa et al. 2003) and affects mainly the sub-Earth latitudes. However, we note that these two types of errors do not change the relative positions of the sub-Earth points for different instances. It is only the error in determination of the rotation period that could significantly affect the computed sub-Earth longitudes at time instances far from our current lightcurve origin. In the following, we will assume that owing to its estimation from a large temporal baseline of 36 years, the current rotation sidereal period of $8.165455 \mathrm{~h}$ can be used to safely span the time interval of the last 3 years when the majority of Lutetia's spectra have been obtained.

\subsection{Spectroscopy}

We obtained five spectra of (21) Lutetia in the range $0.8-2.5 \mu \mathrm{m}$ using the spectrograph SpeX at the 3-m NASA Infrared Telescope Facility (IRTF) located in Mauna Kea, Hawaii.

The observations were performed in remote mode from Centre d'Observation à Distance en Astronomie à Meudon (CODAM) (Birlan et al. 2004) using the low resolution Prism mode $(R=100)$ of the spectrograph. We used a $0.8 \times 15^{\prime \prime}$ slit oriented North-South. The spectra for the asteroid and the solar analog stars were obtained alternatively on two separated locations on the slit denoted A and B (the nodding procedure). The 
Table 1. Physical ephemeris of the asteroid 21 Lutetia. Columns are: UT date for the middle of the exposure interval, planetocentric coordinates (longitude and latitude) for the sub-Solar and sub-Earth points, CCW North Pole angle, phase angle, heliocentric and geocentric distances. We keep the notation from the last column throughout the entire paper.

\begin{tabular}{cccccccccc}
\hline \hline Date $($ UT) & \multicolumn{2}{c}{ SSP $(l, b)\left({ }^{\circ}\right)$} & \multicolumn{2}{c}{ SEP $(l, b)\left({ }^{\circ}\right)$} & North Pole $\left({ }^{\circ}\right)$ & Phase angle $\left({ }^{\circ}\right)$ & $r($ UA) & $\Delta($ UA) & Note \\
\hline $2006 / 03 / 05.410$ & 267.07 & +21.16 & 266.14 & +13.66 & 293.63 & 7.5 & 2.83 & 1.89 & $\mathbf{A}$ \\
$2006 / 03 / 05.525$ & 28.75 & +21.18 & 27.83 & +13.64 & 293.62 & 7.6 & 2.83 & 1.89 & $\mathbf{B}$ \\
$2006 / 04 / 17.227$ & 211.55 & +29.22 & 212.38 & +10.07 & 292.25 & 19.2 & 2.83 & 2.29 & $\mathbf{C}$ \\
$2006 / 04 / 19.208$ & 147.64 & +29.60 & 148.54 & +10.18 & 292.28 & 19.4 & 2.83 & 2.32 & $\mathbf{D}$ \\
$2006 / 04 / 19.215$ & 155.05 & +29.60 & 155.94 & +10.18 & 292.28 & 19.4 & 2.83 & 2.32 & $\mathbf{E}$ \\
\hline $2003 / 03 / 30.432$ & 281.62 & +83.25 & 183.64 & +77.94 & 73.98 & 14.6 & 2.56 & 1.71 & $\mathbf{F}$ \\
$2003 / 03 / 30.479$ & 331.28 & +83.26 & 233.38 & +77.95 & 73.97 & 14.6 & 2.56 & 1.71 & $\mathbf{G}$ \\
$2003 / 03 / 30.568$ & 65.32 & +83.28 & 327.58 & +77.95 & 73.95 & 14.5 & 2.56 & 1.71 & $" 1$ \\
$2003 / 03 / 30.626$ & 126.60 & +83.28 & 28.96 & +77.96 & 73.94 & 14.5 & 2.56 & 1.71 & $\prime$ \\
$2004 / 08 / 12.596$ & 148.96 & -64.48 & 43.41 & -82.66 & 314.47 & 28.3 & 2.12 & 1.73 & $\mathbf{H}$ \\
\hline
\end{tabular}

data reduction process consists in two main steps: 1) obtaining the raw spectra for the object and the solar analog and 2) computation of normalized reflectance spectrum by dividing the asteroid spectrum by the solar analog spectrum and performing a correction for telluric lines.

For the first step, Image Reduction and Analysis Facility (IRAF http: //iraf.noao.edu) was used in conjunction with a script that creates the command files for a specific set of IRAF instructions. Based on some assumptions on the file names, the script will group together the flat field images and those for every individual star or/and asteroids. These command files have the advantage that the reduction procedure should be run only once for the entire data gathered in one night of observations. Careful checking of the results at each step insures the proper procedures are being performed.

The region containing the spectrum is first extracted from the entire $1 \mathrm{k} \times 1 \mathrm{k}$ FITS frame, then a bad-pixel map and a master flat frame are computed to apply the corrections.

Images in the A and B positions of the slit are then paired and subtracted to minimize the sky background and telescope influence. From the subtracted images, each containing a negative and a positive spectrum, the one-dimensional spectra are extracted. Then the wavelength calibration using an argon lamp spectrum is performed. After comparing wavelength scales for the $\mathrm{A}$ and $\mathrm{B}$ beams and computing and correcting for the average shift between the two, the images are trimmed and only the positive half of them is retained and scaled to achieve peak data value.

User defined groups of asteroids and reference stars taken at similar airmasses are combined and single one-dimensional spectra are extracted for each of the groups. Applying the wavelength calibration to all groups' spectra ends the first step of the reduction procedure performed within the IRAF package.

The second step of the data reduction relies on IDL procedures and make use of ATRAN atmospheric model (Lord 1992) to correct for telluric absorption. Final spectra obtained for each group in the previous step, together with their corresponding values of zenith angles are used in this process. Each asteroid spectrum is divided by the spectrum of each reference star to obtain the final normalized reflectance spectrum.

A detailed description of all these steps in data reduction process can be found in Rivkin et al. (2004).

Additionally, cross-checking of the reference star spectra should be performed to examine the data for the possible slope variations in the normalized reflectance spectrum that could artificially induce variations in asteroid's spectra. These effects were previously reported by Hardersen et al. (2006).
Table 2. Circumstances for 2006 observations of (21) Lutetia. Airmass and exposure time for the asteroid and solar analog stars are presented. The label of each spectra is taken from Table 1.

\begin{tabular}{llllll}
\hline \hline Spectrum & \multicolumn{3}{c}{ Asteroid } & \multicolumn{3}{c}{ Solar analog } \\
& $T_{\exp }(\mathrm{s})$ & Airmass & Star & $T_{\exp }(\mathrm{s})$ & Airmass \\
\hline $\mathbf{A}$ & 400 & 1.01 & HD 88371 & 50 & 1.00 \\
$\mathbf{B}$ & 460 & 1.48 & $\prime$ & 60 & 1.41 \\
$\mathbf{C}$ & 330 & 1.02 & HD 87680 & 50 & 1.05 \\
$\mathbf{D}$ & 640 & 1.03 & HD 76332 & 40 & 1.021 \\
$\mathbf{E}$ & 480 & 1.04 & " & 50 & 1.021 \\
\hline
\end{tabular}

The instrumental approach of Vacca et al. (2004) and Rayner et al. (2003) allows to establish a clear relation between the dynamical regime of the NIR detector and its non-linear response. As a consequence, the spectrograph users are strongly advised to operate it in the lower $50 \%$ of the usable detector well depth as long as this region has a quasi-linear response (counts $\leq 4000 \mathrm{DN}$ ). For the non-linearity correction, an iterative procedure was developed and included in the Spextool software package (Cushing et al. 2004). We also used this procedure as a first step to account for non-linearity in our data. Our results prove that the most reliable spectra are obtained when the object and the standard span the same dynamic range of the array. Otherwise spurious variations are seen in the final reflectance spectra (the most affected regions are the shorter $(0.8-1.3 \mu \mathrm{m})$ and longer (2.2-2.5 $\mu \mathrm{m})$ wavelengths).

The ephemeris for physical observations of (21) Lutetia is presented in Table 1 . We also include previously obtained spectra (Birlan et al. 2004, 2006) that were re-reduced and corrected for telluric absorption.

The spectra were acquired under good observing conditions (seeing ranging between $0.7^{\prime \prime}$ and $1.2^{\prime \prime}$ ) with the asteroid and the solar analog stars at similar airmasses (Table 2). The ratio between the spectrum of (21) Lutetia and the solar analog, normalized at $\lambda=1.25 \mu \mathrm{m}$ is presented in Fig. 1 .

\section{Results}

\subsection{Aspects and spectra}

As we can see from Table 1, the aspect of (21) Lutetia varied considerably from one opposition to another. In 2006 the subEarth latitude was around $+10^{\circ}$. At this aspect, we could expect to detect (if present) significant inhomogeneities in asteroid's surface mineralogy by obtaining a series of spectra covering the entire rotational period. Indeed, the sub-Earth latitude indicated a near equatorial aspect of (21) Lutetia. In contrast, during 2003 


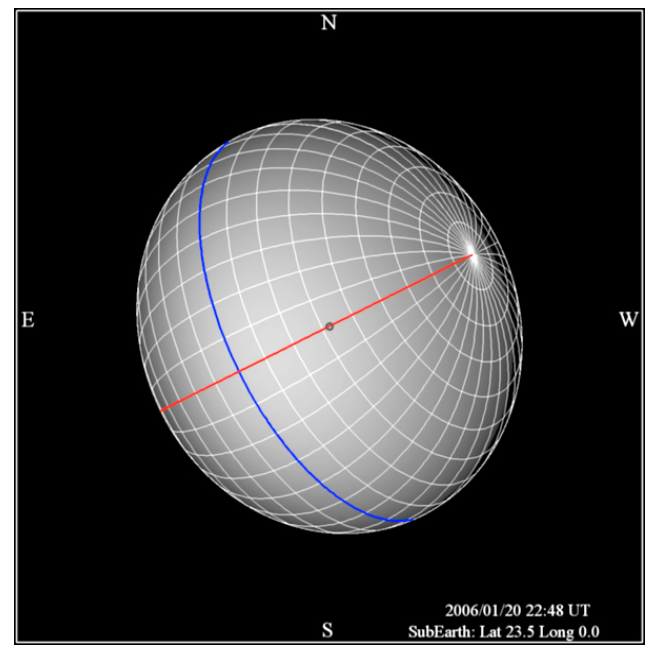

Fig. 2. Aspect of (21) Lutetia at the instant of the first lightcurve minima. Planetocentric prime meridian and the equator are in red and blue respectively.

and 2004 observations, the sub-Earth latitude was $+78^{\circ}$ respectively $-83^{\circ}$ and the spectral information at different rotational phases was collected from almost the same region (northern, and southern hemisphere respectively) of the asteroid. For that reasons in the following we will include in our analysis two out of four spectra from March 2003. The $\mathbf{F}$ and $\mathbf{G}$ spectra from Table 1 are the most reliable for that campaign (lowest airmasses and highest $\mathrm{S} / \mathrm{N}$ ratio) and we consider them as representatives for the northern hemisphere. Correspondingly, southern hemisphere properties are reflected by the 2004 spectrum (H in Table 1).

In addition to the sub-Earth coordinates, other factors should be taken into account when estimating the asteroid surface contributing to a given spectrum. These factors are geometric (shape) and physical ones (scattering laws). During the rotational period, a non-spherical shape of the asteroid will produce variations of the total observable area. The distribution of the incidence and emission angles over this visible area needs to be modeled using a scattering law for the asteroid surface together with the viewing and illumination geometries. For this purpose a triaxial ellipsoid model is a reasonable approach, given the current uncertainties in the pole coordinates.

In order to provide a first estimate of surface area covered by our observations a simplified shape model of (21) Lutetia (Fig. 2) was used (i.e. triaxial ellipsoid with $a / b=1.2, b / c=$ 1.2). To describe the photometric behavior of the asteroid we chose the Lommel-Seeliger scattering law which is based on a simple (i.e. single scattering, isotropic) physical model of diffuse reflection. It accurately describes dark surfaces such as Mercury and the Moon where multiple scattering is almost negligible.

The single scattering contribution of an area element $\mathrm{d} S$ to the total diffuse radiance is given by:

$\mathrm{d} L=\frac{\mu \mu_{0}}{\mu+\mu_{0}} \bar{\omega}_{0} \mathrm{~d} S$

with $\mu, \mu_{0}$ cosine of emission and incidence angle, and $\bar{\omega}_{0}$ the single scattering albedo.

From the brightness distribution over the asteroid's surface we can now estimate the area coming under similar illumination conditions during different nights. In the Fig. 4 we represent the aspect of (21) Lutetia for 3 different epochs: March 2003, August 2004, March 2006. The points with an illumination value greater than $50 \%$ of the point of maximum illumination value,

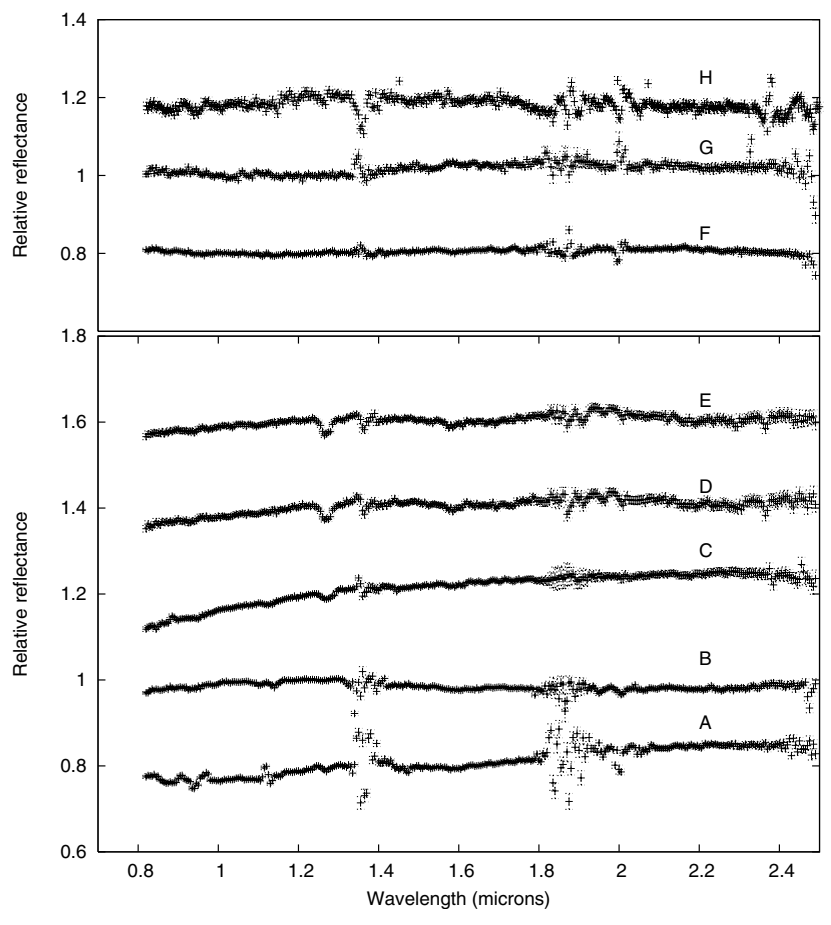

Fig. 3. NIR spectra of (21) Lutetia - March and April 2006 (lower panel), March 2003 and August 2004 (upper panel). Notation from Table 1.

following the scattering law (1), are overplotted. They correspond to a region contributing $98 \%$ to the total diffuse flux.

Figure 5 presents a global picture of the 2006 campaigns with the same areas as described above, in a Mollweide projection of the asteroid surface (assuming an ellipsoid shape model). We also include the areas corresponding to the previous 2003 and 2004 campaigns ( $\mathrm{G}$ and $\mathrm{H}$ respectively).

We see clearly that our observations cover all the equatorial regions (spectra A to $\mathrm{E}$ ) as well as the polar ones ( $\mathrm{F}$ to $\mathrm{H}$ ). A and $\mathbf{B}$ regions were observed in March 2006 while $\mathbf{C}, \mathbf{D}$, and $\mathbf{E}$ correspond to the April 2006 observations. The spectra for each of these regions is represented in the lower panel of Fig. 3 (identified by the same letters as in Table 1). The 2006 spectra are neutral and featureless. However we can distinguish a slightly positive slope for the $\mathbf{A}$ and $\mathbf{C}$ spectra. According to Fig. 5 these spectra correspond to overlapping regions of the asteroid surface. The $\mathbf{C}$ spectrum was obtained one month apart from the A spectrum. The slight differences between these spectra while the reflected surface is partially the same prove the robustness of the reduction procedure. The $\mathbf{B}$ spectrum is the most neutral one and it corresponds to a region far from $\mathbf{A}$ and $\mathbf{C}$ (sub-Earth longitude difference around $180^{\circ}$ ). The $\mathbf{E}$ and $\mathbf{D}$ spectra are taken from almost the same surface of the asteroid. They are similar, and show a slope intermediate between $\mathbf{C}$ and $\mathbf{B}$ spectra. A quantitative analysis of similarites among spectra is presented further in Fig. 7.

\subsection{Comparison with laboratory spectra}

One of the common methods of investigation for atmosphereless body surfaces is the comparative mineralogy. Our objective is to find the closest analog of the obtained spectra among spectra 

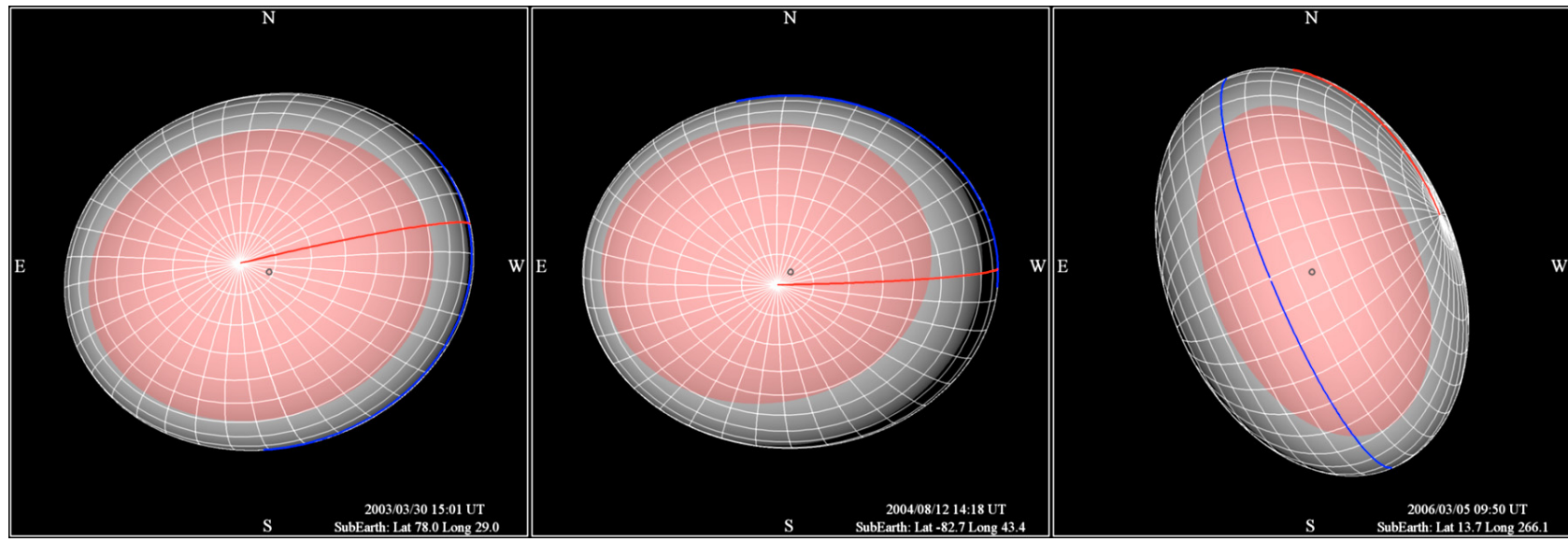

Fig. 4. (21) Lutetia aspect for March 2003 (left), August 2004 (center), and March 2006 (right). The scattering properties of the ellipsoid surface are described by Lommel-Seeliger's law (1). Note the effect of high phase angle (28.3 ${ }^{\circ}$ for 2004 observations. The points with an illumination value greater than $50 \%$ of the point of maximum illumination value, following the scattering law (1), are overplotted. The March 2003 aspect is a pole-on geometry of the northern hemisphere while the observations of August 2004 are a pole-on geometry of the southern hemisphere. In 2006 the observed aspect of the asteroid was nearly equatorial. Planetocentric equator (in blue) and prime meridian (in red) are drawn.

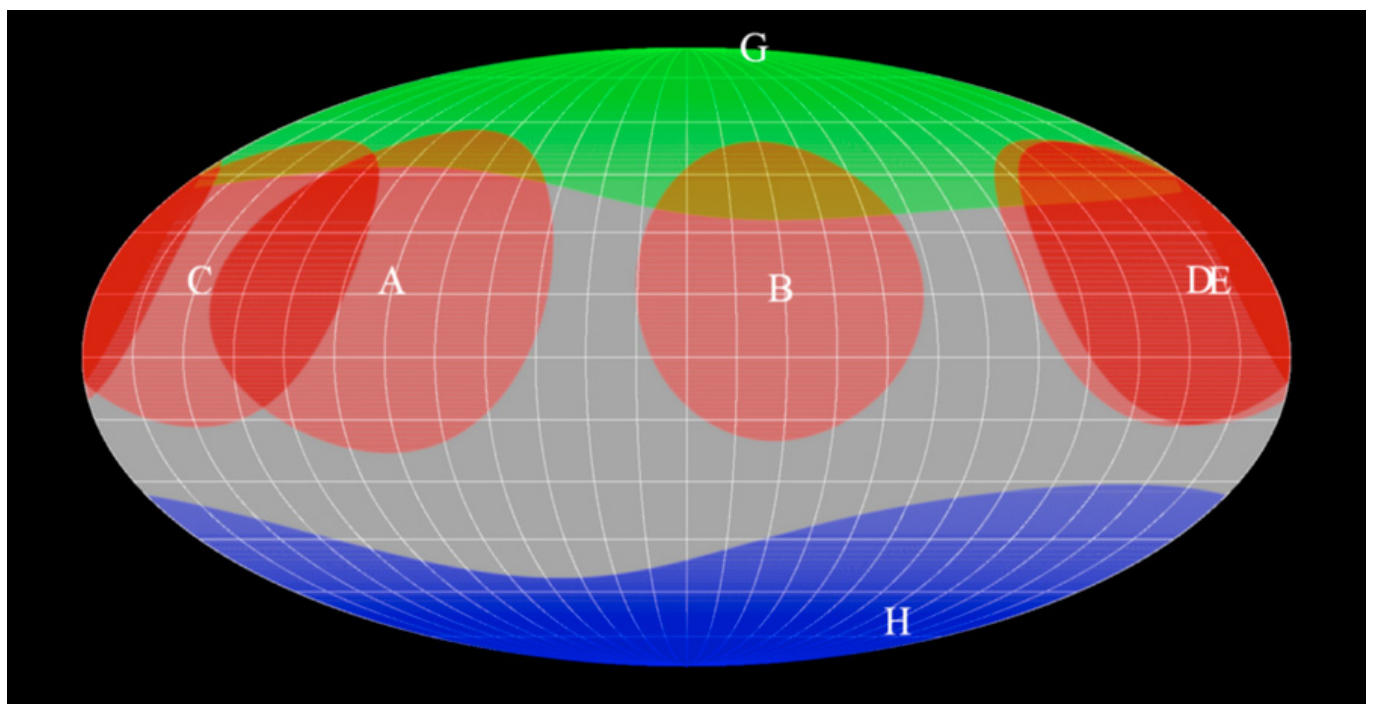

Fig. 5. Mollweide projection of the asteroid surface assuming an ellipsoid shape model. The regions observed in 2006 following the same approach as in Fig. 4 are overplotted in red and the regions observed in 2003 and 2004 are in green and blue respectively (G, H). All the areas are marked with the designated letter from Table 1. The reflecting surface corresponding to the $\mathbf{B}$ spectrum is distinct from those of $\mathbf{A}$ and $\mathbf{C}$ one. We note that the most distinct spectra, $\mathbf{B}$ and $\mathbf{C}$ are on the opposite sides of the asteroid (sub-Earth longitudes difference around $180^{\circ}$ ). We interpret the corresponding regions of $\mathbf{D}$ and $\mathbf{E}$ spectra as a transitional surface (mineralogy) between the $\mathbf{B}$ and $\mathbf{C}$ regions. From Fig. 3 we see that regions far from $\mathbf{C}$ tend to exhibit spectra with more neutral slope.

of representatives for meteoritic classes taken from RELAB database. The $\chi^{2}$ test with the following definition was used

$\chi^{2}=\frac{1}{N_{w}} \sum_{i=1}^{N_{w}} \frac{\left(R_{i}-f\left(w_{i}\right)\right)^{2}}{f\left(w_{i}\right)}$

where $N_{w}$ is the number of $R_{i}$ reflectance values at $w_{i}$ wavelength from the meteorite spectra, and $f\left(w_{i}\right)$ the value of 12 th degree polynomial fitting the visible+NIR (VNIR) spectrum of Lutetia. The VNIR spectra were constructed by means of visible spectra obtained by Barucci et al. (2005) using their overlapping region $(0.82-0.95 \mu \mathrm{m})$. Our choice for the above V spectrum was based on its similarities with previously published ones (Bus 1999; Carvano et al. 2003). This approach may imply that a possible spectral variation in the visible domain is less important than those in the NIR one.
In order to keep a homogeneity of the meteoritic spectra sample, from the RELAB database we extracted all the spectra obtained with the integrating sphere spectrometer (Gaffey 1976). This dataset is considered to be the most consistent and comprehensive among the meteorite spectra (Britt et al. 1992). The only exception was made for the iron meteorite Babb's Mill spectrum. This sample preparation condition (clean cut fresh surface) seems to be inappropriate for studying the asteroidmeteorite connection (Britt \& Pieters 1988). Indeed this sample has the most flat spectrum in the VNIR domain (slope $=0.118$ ) as compared with the other metallic meteorites spectra (slope = $0.367 \pm 0.060$ ). As expected, the $\chi^{2}$ test finds for different spectra of (21) Lutetia a different analog in the meteorites spectra. The results are plotted in Fig. 6 while the values of $\chi^{2}$ and the meteorite references are presented in Table 3 .

However direct comparison between laboratory spectra of meteorites and asteroid spectra must be carefully interpreted. 
Table 3. The best fit for (21) Lutetia's spectra compared with the selected subset from the RELAB database. The table presents the letter designation for Lutetia's spectrum, the meteorite and its type and the $\chi^{2}$ result. Identical names for the meteorite indicate different sample.

\begin{tabular}{cllc}
\hline \hline Spectrum & Meteorite & Type & $\chi^{2}\left(\times 10^{6}\right)$ \\
\hline $\mathbf{A}$ & Hvittis & EL6 & 416 \\
& Hvittis & EL6 & 504 \\
& Pillistfer & EL6 & 629 \\
& Sevrukovo & L5 & 633 \\
& Kainsaz & CO3 & 860 \\
\hline $\mathbf{B}$ & Orgueil & CI1 & 599 \\
& Orgueil & CI1 & 656 \\
& Grosnaja & CV3 & 742 \\
& Vigarano & CV3 & 1015 \\
& Kainsaz & CO3 & 1355 \\
\hline $\mathbf{C}$ & Hvittis & EL6 & 460 \\
& Hvittis & EL6 & 471 \\
& Pillistfer & EL6 & 624 \\
& St. Mark's & EH5 & 768 \\
& Khairpur & EL6 & 840 \\
\hline $\mathbf{D}$ & Orgueil & CI1 & 749 \\
& Orgueil & CI1 & 774 \\
& Kainsaz & CO3 & 1046 \\
& Vigarano & CV3 & 1137 \\
& Hvittis & EL6 & 1180 \\
\hline $\mathbf{E}$ & Orgueil & CI1 & 684 \\
& Orgueil & CI1 & 720 \\
& Vigarano & CV3 & 854 \\
& Kainsaz & CO3 & 897 \\
& Grosanaja & CV3 & 1008 \\
\hline $\mathbf{F}$ & Vigarano & CV3 & 319 \\
& Kainsaz & CO3 & 533 \\
& Grosnaja & CV3 & 559 \\
& Felix & CO3 & 687 \\
& Warrenton & CO3 & 936 \\
\hline H & Vigarano & CV3 & 530 \\
& Kainsaz & CO3 & 576 \\
& Grosnaja & CV3 & 1003 \\
& Felix & CO3 & 1088 \\
& Warrenton & CO3 & 1237 \\
\hline & Orgueil & CI1 & 460 \\
& Orgueil & CI1 & 515 \\
& Grosnaja & CV3 & 721 \\
& Vigarano & CV3 & 1071 \\
Kainsaz & CO3 & 1336 \\
\hline & & & \\
& &
\end{tabular}

In this situation, the $\chi^{2}$ statistical test is sensitive to several parameters (Pieters \& McFadden 1994). This $\chi^{2}$ test evaluates with equal weight the entire wavelength interval and it is not so sensitive to subtle features as shallow absorbtion bands. The $\chi^{2}$ values from Table 3 should be interpreted as the most probable mineralogy analog to the (21) Lutetia surface. The homogeneity in the set of the best fits should be seen as a strong indicator of a comparable mineralogy. For instance the $\mathbf{C}$ spectrum of Lutetia fits the bulk collection of chondritic enstatite meteorites very well while the $\mathbf{H}$ spectrum fits the CI, CV carbonaceous chondrite.

\subsection{Discussion}

Our NIR spectra are in agreement with the previously published ones. The spectra are neutral in color and reveal no major absorption features.

In the equatorial aspect presented by (21) Lutetia during the 2006 observations, we distinguish a global slope variation over the rotational period. On the basis of the physical ephemeris we

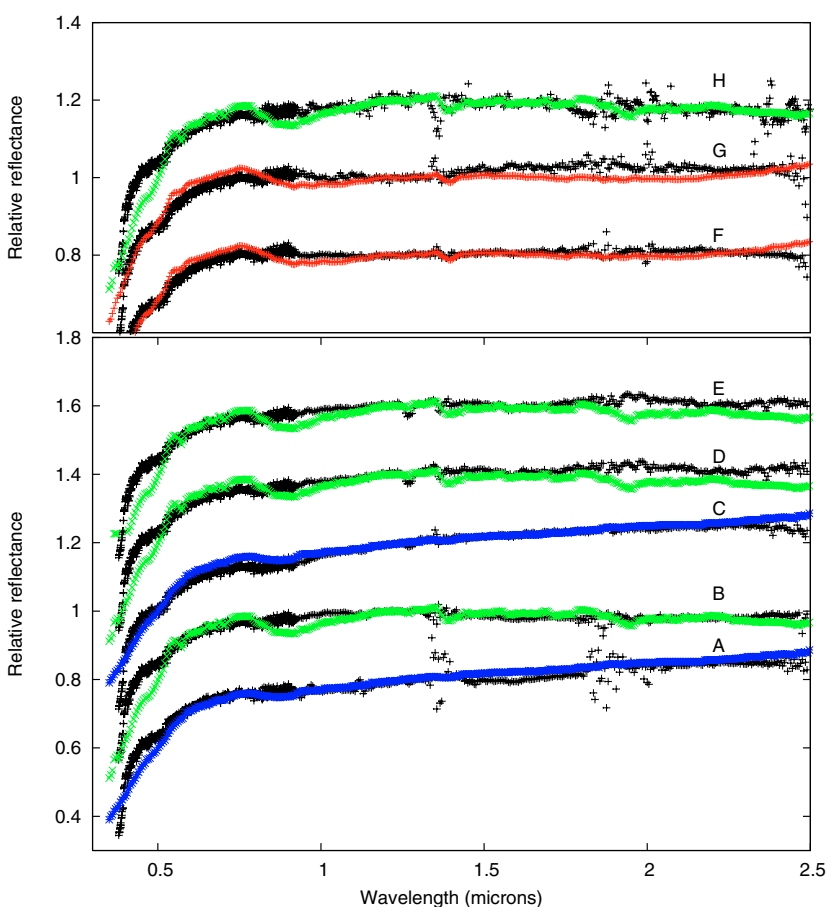

Fig. 6. VNIR spectra of (21) Lutetia from Fig. 2. The corresponding matching spectra indicated by the $\chi^{2}$ test are overplotted. Notation from Table 1. The spectra for the best mineral analogs are superimposed: Hvittis E6 in blue, Orgueil CI in light green, and Vigarano CV3 in red.

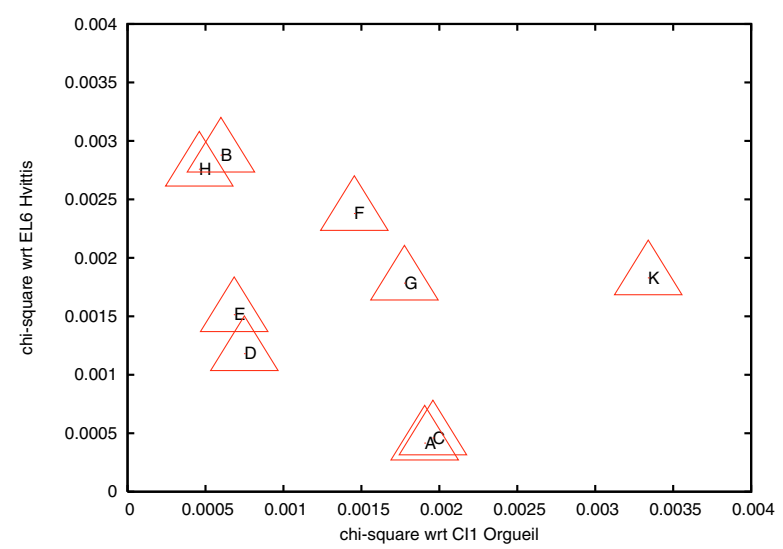

Fig. 7. $\chi^{2}$-space of the $\chi^{2}$ coefficients between our spectra and the end members of the oxidized-reduced range: CI1 Orgueil and EL6 Hvittis. $\mathrm{K}$ label denote the spectrum of Kaidun

prefer the interpretation of a real mineralogical variation over an instrumentation induced artifact. The $\chi^{2}$ test performed in Sect. 3.2 allows us to infer the most probable meteoritic analog as presented in Table 3. As we can see, this table shows quite a large variety of meteoritic analogs covering a wide lithology. However, as long as all the mineralogical classes of the inferred analogs are chondritic we are in agreement with a primitive nature of (21) Lutetia's surface.

The predominant type of meteoritic analogs are the carbonaceous chondrite CI, CV (Orgueil, Grosnaja and Vigarano principally). For the $\mathbf{A}$ and $\mathbf{C}$ spectra the meteoritic analogs are the highly reduced enstatite chondrite E5, E6. Usually CI, CV meteorites exhibit various degrees of oxidation. CI meteorite Orgueil shows signs of extensive aqueous alteration being devoid of chondrules that are most probably destroyed during this process. The most abundant mineral phases in Orgueil are magnetites and 
carbonates with the latter formed in the very early stages of aqueous alteration on the CI parent body (Endress et al. 1996). These carbonates are believed to have precipitated from aqueous solution circulating on the CI parent body (Endress \& Bischoff 1995).

Grosnaja belongs to the oxidized group of the CV3 meteorites. It has undergone some aqueous alteration, which has resulted in the formation of intimately intergrown phyllosilicates in the matrix and in the chondrules (Keller \& McKay 1993). The lithological studies of CV3 meteorites further divide this oxidized group into two distinct subgroups (Krot et al. 1998). Based on the matrix abundance, metal to magnetite ratio, phylosillicates and pure fayalite presence, Grosnaja belongs to the $\mathrm{CV}_{\text {oxB }}$ (Krot et al. 1998).

Vigarano is a meteorite of the reduced group of the CV3 chondrites and experienced some very mild aqueous alteration (Lee et al. 1996). Its texture and mineralogy is mainly comprised by chondrules (up to $50 \%$ of the total matter in the CV type), CAIs, chondrite fragments, as well as metal and sulfide grains.

The meteorites containing enstatitic chondrites are nondifferentiated. They consist mainly of nearly FeO-free enstatite, variable amounts of metallic $\mathrm{Fe}, \mathrm{Ni}$, troilite, and rare minerals formed under heavy-reducing conditions. Depending on the iron abundance we can distinguish EL group with $\mathrm{Fe} \leq 12 \%$ and $\mathrm{EH}$ group with Fe content larger than $15 \%$. The highly reduced nature of typical enstatite-rich meteorites suggests that aqueous alteration was an improbable process (Keil 1989).

One of the key questions concerning possible links between meteoritic classes and (21) Lutetia is the following:

"How can such different lithologies and apparently incompatible minerals be present on the surface of the asteroid?" In other words, the best fit between the (21) Lutetia's spectra and meteoritic interpretations must be explained in terms of the coexistence of mineralogies experiencing different origins and evolution. Such an image of (21) Lutetia would be supported if meteorites exhibiting "strange" collection of mineralogies, linked with different evolution scenarios (oxidized - reduced, aqueous altered - unaltered) are present in the terrestrial collections.

One of the objects consistent with this scenario is the extremely heterogeneous meteorite Kaidun (Zolensky \& Ivanov 2003). It was studied during the last two decades and it revealed a mixture of "incompatible" types of meteoritic materials, principally carbonaceous and enstatite chondrite (EH, EL, CV, CM), corresponding to the most oxidized and the most reduced meteorites. Oxygen isotopic data for many samples of Kaidun are intermediate between the results for E, CI, CV, and CM chondrites. This implies that most of the analyzed samples are actually an intimate mixture of this chondritic types. Additionally these mineralogies exhibit a complete range of aqueous alteration ranging from completely altered materials to the anhydrous ones (Zolensky 2005). In contrast to all other known enstatite chondrite meteorites, hydrated phases are abundant in all EH lithologies of Kaidun. The high heterogeneity of Kaidun was then explained by the formation of its parent body from materials originated from different asteroids (E, D, C). Various origins have been proposed for Kaidun: a carbonaceous chondrite like asteroids in a high eccentricity orbit (Ivanov 1997), a large carbonaceous chondrite body like Ceres (Ivanov et al. 2001), and the Mars satellite Phobos (Zolensky 2005).

In Fig. 7 we plot the $\chi^{2}$ coefficients between our spectra and the end members of the oxidized-reduced range: CI1 Orgueil and EL6 Hvittis. As we can see there is a strong correlation between the spectral feature, location on the asteroid surface, and their positions in the above " $\chi^{2}$-space". Both of our A and $\mathbf{C}$ spectra show a good agreement with the reduced class of selected meteorites while $\mathbf{H}$ and $\mathbf{B}$ are a good match for the highly aqueous altered carbonaceous chondrite Orgueil. The D and E spectra (regions) as well as $\mathbf{F}$ and $\mathbf{G}$ are interpreted as transitional surfaces (mineralogies).

We place in Fig. 7 the only Kaidun spectrum found in the literature (Zolensky \& Ivanov 2003). Its position (label K) reveals a good match with our spectra.

A primitive, heavily aqueous altered carbonaceous chondrite composition for some M-type asteroids was previously suggested by Vilas (1994). A spectrophotometric survey of M- and E-type asteroids by Rivkin et al. (1995) revealed the presence of hydrated minerals on the surface of two M-type asteroids and established a new W (wet) class based on the presence of the $3 \mu \mathrm{m}$ feature diagnostic for structural hydroxyl $(\mathrm{OH})$ and interlayer and adsorbed water. In a recent survey (Rivkin et al. 2000) new members (including (21) Lutetia) were added to this new W-class. A statistical analysis found a significant correlation between the diameters of M-type asteroids and the presence of hydration signatures. Large M-type asteroids $(D \geq 65 \mathrm{~km})$ are likely to be hydrated while the smaller ones seem to be entirely anhydrous. This W-class and the hydrated E asteroids may be mixtures of high-albedo anhydrous minerals with a possibly primitive, hydrated minerals from low-albedo source. Rivkin et al. (2000) concluded that (21) Lutetia may be akin to enstatite chondrite or salt-rich carbonaceous chondrites despite the mismatch in thermal albedo.

Is it possible to reconcile between the inferred primitive nature of (21) Lutetia and its high thermal albedo?

Since pure enstatite $\left(\mathrm{MgSiO}_{3}\right)$ is spectrally neutral, very white, with albedo value typically greater than 0.5 one of the explanations could be its confirmed presence. A limited areal coverage of this material could raise the albedo of (21) Lutetia without hiding the carbonaceous chondrite-like spectrum.

Another explanation for the currently observed value of Lutetia's albedo $(0.208 \pm 0.025$ deduced by Mueller et al. 2006) is given by the aqueous alteration process that should slowly increase the albedo as it progress. Continued aqueous alteration will eventually remove the iron from phyllosilicates and sequester it in magnetite and iron sulfide. Accordingly, the depth of the $0.7 \mu \mathrm{m}$ feature due to a $\mathrm{Fe}^{2+}-\mathrm{Fe}^{3+}$ charge transfer band in iron-bearing phyllosilcates will be reduced leaving only the $3 \mu \mathrm{m}$ band as a signature of hydration (Vilas 1994).

Last but not least, if the bright sulphate and/or carbonate veins present in CI chondrites (Tomeoka 1990) were reaching the asteroid surface, they could form high reflectance "patches" (however, it was suggested, Gounelle \& Zolensky 2001, that sulphate veins in five CI1 meteorites are of terrestrial origin). Isotopic oxygen fractionation data for CI, CV and CM meteorites (Young et al. 1999) requires, for aqueous alteration in the parent body, a flow of reactive water down a temperature gradient. Following the authors experiment, the alteration occurs more strongly deeper into the parent body, with external layers less altered. For increasing the albedo, this hypothesis needs post-aqueous alteration resurfacing via subsequent collision to expose brighter deeper layers. However they estimated that a real heterogeneous body could concentrate up to $10 \%$ of altered material into highly altered areas separated by alteration free areas.

We should not exclude that all these three scenarios might have ocurred together to raise the albedo. Is worth noting that the heterogeneity implied in these scenarios would necessarily produce variations in the asteroid spectra over the rotational phase.

One of the key questions in solving the problems concerning (21) Lutetia (and more generally the mineralogical interpretation 
of asteroids spectra) is the scale factor of the analysis (micron to $\mathrm{cm}$-size of laboratory samples versus $\mathrm{km}$ size for asteroids). This is a wide subject and unsolvable for the moment by groundbased investigation. The relation between Kaidun meteorite lithologies and Lutetia is considered only as a proof of the coexistence of various mineralogies even at $\mathrm{cm}$-scale.

(21) Lutetia has a long record of producing puzzling results. The first hint of its spectral variations dates back to the ECAS program (Zellner et al. 1985). From these results Vilas (1994) found spatial variations in the presence of water of hydration in the asteroid's surface material but: "...the result for the $M$ class asteroids 21 remains unexplained. Insufficient data exist at this time to address in depth the question of spatial variability in water of hydration across asteroid surfaces". Our results add strong evidence for the intrinsic reality of this variation on the surface of (21) Lutetia.

\section{Conclusions}

We have presented five new NIR spectra of (21) Lutetia obtained in March and April 2006 using SpeX/IRTF in remote observing mode from Meudon, France. From the latest photometric observations of the asteroid we have constructed the ephemeris for physical observations. Due to a near-equatorial aspect of the asteroid, we have sampled almost all the asteroid's surface during the rotational phase.

The spectra revealed no major absorption features. A clear spectral variation was observed, and a good correlation between the spectra and the rotational phase and geometric aspect was found. Two of the most different spectra we have obtained correspond to two opposite sides of the asteroid (sub-Earth longitude difference around $180^{\circ}$ ).

A comparison with laboratory spectra of some representative for the meteoritic classes was performed using a $\chi^{2}$ fitting test. For the neutral spectra a carbonaceous chondrite yielded the best fit while for spectra with a slight positive slope the enstatitic chondrite was a good match. Thus, our analysis suggests a primitive, chondritic nature of (21) Lutetia. Differences in the spectra are interpreted in term of coexistence of several lithologies on the surface where aqueous alteration may have played an important role.

Acknowledgements. We thank Dr. Andrew Rivkin for useful comments which improved the article.

This research utilizes spectra acquired by Hiroi, Pieters, and Gaffey, with the NASA RELAB facility at Brown University.

The article is based on observations acquired with IRTF as well asa the CODAM remote facilities, and the $105 \mathrm{~cm}$ telescope at Pic du Midi, France. We thank all the telescope operators for their contribution. The work of Alin Nedelcu was supported by ESA traineeship programme.

Agnieszka Kryszczynska was supported by the Polish Grant 1 P03D 008 26. The photometric observations were reduced with the CCLRS STARLINK package. We acknowledge Antonella Barucci and Marcello Fulchignoni for useful discussions.

\section{References}

Barucci, M. A., Capria, M. T., Coradini, A., \& Fulchignoni, M. 1987, Icarus, 72, 304

Barucci, M. A., Fulchignoni, M., Fornasier, S., et al. 2005, A\&A, 430, 313

Birlan, M., Barucci, M. A., Vernazza, P., et al. 2004, New Astron., 9, 343

Birlan, M., Vernazza, P., Fulchignoni, M., et al. 2006, A\&A, 454, 677

Britt, D. T., \& Pieters, C. M. 1988, in Lunar and Planetary Science Conference, ed. G. Ryder, 503

Britt, D. T., Tholen, D. J., Bell, J. F., \& Pieters, C. M. 1992, Icarus, 99, 153

Bus, S. J. 1999, Ph.D. Thesis

Bus, S. J., \& Binzel, R. P. 2002, Icarus, 158, 146

Carvano, J. M., Mothé-Diniz, T., \& Lazzaro, D. 2003, Icarus, 161, 356

Cushing, M. C., Vacca, W. D., \& Rayner, J. T. 2004, PASP, 116, 362

Dotto, E., Barucci, M. A., Fulchignoni, M., et al. 1992, A\&AS, 95, 195

Endress, M., \& Bischoff, A. 1995, in Lunar and Planetary Institute Conference Abstracts, 371

Endress, M., Zinner, E., \& Bischoff, A. 1996, Nature, 379, 701

Gaffey, M. J. 1976, J. Geophys. Res., 81, 905

Gounelle, M. \& Zolensky, M. E. 2001, Meteor. Planet. Sci., 36, 1321

Hardersen, P. S., Gaffey, M. J., Cloutis, E. A., Abell, P. A., \& Reddy, V. 2006, Icarus, 181, 94

Howell, E. S., Merenyi, E., \& Lebofsky, L. A. 1994, J. Geophys. Res., 99, 10847 Ivanov, A. V. 1997, Meteor. Planet. Sci., 32, 65

Ivanov, A. V., Zolensky, M. E., Kononkova, N. N., Yang, S. V., \& Migdisova, L. F. 2001, Meteor. Planet. Sci. 36, 87

Keil, K. 1989, Meteoritics, 24, 195

Keller, L. P., \& McKay, D. S. 1993, Meteoritics, 28, 378

Krot, A. N., Petaev, M. I., Scott, E. R. D., et al. 1998, Meteor. Planet. Sci., 33, 1065

Lagerkvist, C.-I., Erikson, A., Debehogne, H., et al. 1995, A\&AS, 113, 115

Lazzarin, M., Marchi, S., Magrin, S., \& Barbieri, C. 2004, A\&A, 425, L25

Lee, M. R., Hutchinson, R., \& Graham, A. L. 1996, Meteor. Planet. Sci., 31, 477

Lord, M. M. 1992, Technical Report, JPL

Lupishko, D. F., \& Mohamed, R. A. 1996, Icarus, 119, 209

Lupishko, D. F., \& Velichko, F. P. 1987, Kinematika i Fizika Nebesnykh Tel, 3, 57

Magri, C., Ostro, S. J., Rosema, K. D., et al. 1999, Icarus, 140, 379

Mueller, M., Harris, A. W., Bus, S. J., et al. 2006, A\&A, 447, 1153

Pieters, C. M., \& McFadden, L. A. 1994, Ann. Rev. Earth Planet. Sci., 22, 457

Prokof'eva, V. V., Bochkov, V. V., \& Busarev, V. V. 2005, Sol. Sys. Res., 39, 410

Rayner, J. T., Toomey, D. W., Onaka, P. M., et al. 2003, PASP, 115, 362

Rivkin, A. S., Howell, E. S., Britt, D. T., et al. 1995, Icarus, 117, 90

Rivkin, A. S., Howell, E. S., Lebofsky, L. A., Clark, B. E., \& Britt, D. T. 2000, Icarus, 145, 351

Rivkin, A. S., Binzel, R. P., Sunshine, J., et al. 2004, Icarus, 172, 408

Tedesco, E. F., Veeder, G. J., Fowler, J. W., \& Chillemi, J. R. 1992, The IRAS Minor Planet Survey, Tech. rep.

Tholen, D. J. 1989, in Asteroids II, ed. R. P. Binzel, T. Gehrels, \& M. S. Matthews, 1139

Tomeoka, K. 1990, Nature, 345, 138

Torppa, J., Kaasalainen, M., Michalowski, T., et al. 2003, Icarus, 164, 346

Vacca, W. D., Cushing, M. C., \& Rayner, J. T. 2004, PASP, 116, 352

Vilas, F. 1994, Icarus, 111, 456

Young, D. E., Ash, D. R., \& Rumble, D. 1999, Science, 286, 1331

Zappala, V., di Martino, M., Knezevic, Z., \& Djurasevic, G. 1984, A\&A, 130, 208

Zellner, B., Lebertre, T., \& Day, K. 1977, in Lunar and Planetary Science Conference, ed. R. B. Merril, 1111

Zellner, B., Tholen, D. J., \& Tedesco, E. F. 1985, Icarus, 61, 355

Zolensky, M. E. 2005, LPI Contributions, 1267, 32

Zolensky, M. E., \& Ivanov, A. 2003, Chemie der Erde/Geochemistry, 63, 185 